\title{
Indução do estro em novilhas Nelore com implante intravaginal de progesterona de quarto uso*
}

\section{Induction of estrus in Nelore heifers with intravaginal progesterone implant used forth time}

\author{
Jakeline Fernandes Cabral, ${ }^{* *}$ Karen Martins Leão, ${ }^{* * *}$ Marco Antônio Pereira da Silva, ${ }^{* \star *}$ Rafaella Belchior Brasil ${ }^{* *}$
}

\begin{abstract}
Resumo
Objetivou-se neste estudo avaliar a indução do estro em novilhas Nelore, utilizando implante intravaginal de progesterona de quarto uso. Em duas fazendas comerciais (Grupos I e II), foi utilizado um total de 260 novilhas ( $n=117$ e 143; grupos I e II, respectivamente), da raça Nelore (Bos taurus indicus) que não apresentaram sinais de estro nos dois primeiros meses da estação de monta. Nos dois grupos, o estro foi induzido de acordo com o seguinte protocolo: inserção de um implante intravaginal de progesterona de quarto uso (D0) associado a aplicação de $2,0 \mathrm{mg}$ de benzoato de estradiol (BE).

No momento da retirada do implante (D8), uma segunda dose de BE $(1,0 \mathrm{mg})$ foi administrada. Após a retirada do implante, a observação do cio foi realizada, com auxílio de rufiões (relação 1:20), a cada 12 horas durante 15 dias. Neste período, os animais em estro foram artificialmente inseminados. Após este período, as fêmeas não detectadas em estro foram colocadas com touros de repasse (relação 1:50). O diagnóstico de gestação foi realizado por palpação retal cinco meses após a inseminação artificial. A porcentagem de animais em estro e a taxa de fertilidade nos dois grupos foram comparadas pelo teste de qui-quadrado. Nos dois grupos de animais, a porcentagem de animais em estro não foi diferente (70,94\% e 60,84\%; grupos I e II, respectivamente). Nos dois grupos, a deteç̧ão de animais em estro ficou concentrada nos dois primeiros dias após a retirada do implante intravaginal ( $79 \%$ e $82 \%$, grupos I e II, respectivamente). A taxa de fertilidade também não diferiu entre os grupos (66,67\% e $57,34 \%$; grupos I e II, respectivamente). Conclui-se que a indução de estro em novilhas Nelore utilizando o implante intravaginal de progesterona de quarto uso foi eficaz, considerando o número de animais em cio após o término do protocolo.
\end{abstract}

Palavras-chave: Bos taurus indicus, estro, inseminação artificial.

\begin{abstract}
The aim of the present study was to evaluate the induction of estrus in Nelore (Bos taurus indicus) heifers using intravaginal progesterone implant used for the fourth time. In two different commercial farms (Groups I and II), 260 Nelore heifers ( $\mathrm{n}=117$ and 143, groups I and II; respectively) that did not showed estrus behavior in the first two months of the breeding season were used. In both groups, estrus induction was performed according to the following protocol: an intravaginal progesterone implant used for the forth time was inserted (D0), at the same time $2.0 \mathrm{mg}$ of estradiol benzoate (EB) was given intramuscularly. At the moment of intravaginal implant withdrawal (D8), a second dose $(1.0 \mathrm{mg})$ of EB was administered. After the implant removal, estrus detection was performed, with the assistance of bull teasers (1:20 ratio), every 12 hours during 15 days. During this period, animals that showed estrus behavior was artificially inseminated. Those heifers that did not showed estrus behavior after 15 days were placed with fertile bulls (1:50 ratio). Pregnancy diagnosis was performed by rectal palpation five months after artificial insemination. Percentage of heifers in estrus and fertility rate were compared between groups by qui-square test. In both groups of animals, percentage of animals in estrus was not different $(70.94 \%$ and $60.84 \%$, group I and II, respectively). In both groups, estrus detection was concentrated in the first two days after intravaginal implant withdrawal ( $79 \%$ and $82 \%$, group I and II, respectively)., The fertility rate was not different between groups $(66.67 \%$ and $57.34 \%$, group I and II, respectively) In conclusion, estrus induction of Nelore heifers using intravaginal progesterone implant used for the forth time was effective, considering the number of animals in estrus after the protocol.
\end{abstract}

Keywords: Bos taurus indicus, estrus, artificial insemination.

\footnotetext{
* Recebido em 10 de abril de 2012 e aceito em 14 de fevereiro de 2013.

** Mestranda do Programa de Pós-graduação em Zootecnia - Instituto Federal de Educação Ciência e Tecnologia Goiano - Campus Rio Verde.

*** Docente do Programa de Pós-graduação em Zootecnia - Instituto Federal de Educação Ciência e Tecnologia Goiano - Campus Rio Verde.

Autor para correspondência: e-mail: Karen Martins Leão. E-mail: karenleao2@yahoo.com.br.
} 


\section{Introdução}

Mesmo com um rebanho numeroso com aproximadamente 209,5 milhões de bovinos (BRASIL, 2011), a baixa produtividade e a concorrência com outras fontes de origem animal têm sido um desafio para a pecuária brasileira.

Assim, novas biotecnologias têm sido aplicadas na reprodução de bovinos para que aumente a produtividade do rebanho nacional. A inseminação artificial (IA) é uma das tecnologias mais utilizadas nos rebanhos brasileiros e consiste na deposição do sêmem no aparelho reprodutivo da fêmea com auxílio de equipamentos apropriados (Marques et al., 2008).

A IA possui várias vantagens, como dispor para o produtor menor custo por concepção em relação à monta natural, melhoramento genético, acasalamento corretivo, cruzamento entre raças, prevenção de acidentes com as fêmeas, aumento de número de descendentes de um bom reprodutor, controle zootécnico do rebanho, padronização do rebanho, uso de touros após a morte, melhor controle sanitário da reprodução (Morais et al., 2005).

Embora a IA apresente diversas vantagens, apenas uma pequena porção das fêmeas no Brasil são inseminadas, correspondendo a $7 \%$ das fêmeas em idade reprodutiva (Almeida, 2010).

De acordo com Moreira et al. (2007), as limitações do uso da IA em fêmeas bovinas de corte incluem falhas na detecção de cios, grande extensão das propriedades rurais e mão de obra pouco qualificada. Uma das formas para se solucionar tais problemas é a utilização de protocolos de sincronização do ciclo estral e ovulação que permitam realizar a IA com horário predeterminado, ou seja, sem a necessidade da detecção do cio. O emprego de tais protocolos dispensa a observação de estros e possibilita a realização da inseminação artificial em tempo fixo (IATF), sendo que os fármacos utilizados para promover tais eventos fisiológicos em programas de IATF devem oferecer uma vantajosa relação custo/benefício (Almeida et al., 2006).

Os protocolos hormonais utilizados para a IATF são capazes de induzir a ciclicidade em vacas em anestro (Baruselli et al., 2003). De acordo com Azeredo et al. (2007), a utilização de protocolos hormonais em novilhas com intuito de induzir e sincronizar o estro, teve resultados positivos, pois a P4 quando utilizada em novilhas, inclusive pré-púberes, é capaz de iniciar a atividade ovariana cíclica.

Em função dos custos dos implantes intravaginais de progesterona, a reutilização destes implantes nos programas de IATF tem como objetivo alcançar uma melhor relação custo/beneficio. Porém, tanto em vacas quanto em novilhas, os resultados de reutilização dos implantes de P4 possuem resultados variados e de acordo com Pinto-Neto et al. (2009), são diversos fatores que podem afetar os resultados como raça, resposta individual, estado nutricional, alimentação, combinação hormonal adotada.

Baruselli et al. (2006) observaram que a reutilização de implantes intravaginais é uma prática que reduz custos de um programa de indução e sincronização do estro e ovulação na espécie bovina, e vem contribuindo para resultados expressivos de fertilidade.

Com o presente estudo objetivou-se avaliar a indução do estro em novilhas Nelore, utilizando implante intravaginal de progesterona de quarto uso.

\section{Material e métodos}

O estudo foi realizado na Agropecuária Baumgart - Fazendas Reunidas localizadas nos municípios de Rio Verde (Fazenda I) e Paraúna (Fazenda II) no estado de Goiás, no período de 5 de janeiro a 8 de fevereiro de 2011.

Com o objetivo de avaliar o efeito da utilização de implantes intravaginais de progesterona de quarto uso sobre a indução de estro, foram utilizadas 260 novilhas comerciais da raça Nelore (Bos taurus indicus) que não foram observadas em estro nos dois primeiros meses de estação de monta.

O índice pluviométrico da Propriedade I (Rio Verde) foi de 1.769 $\mathrm{mm}$ ao ano e na Propriedade II (Paraúna) foi de $1.820 \mathrm{~mm}$ ao ano. Na fazenda II o período da entressafra que antecedeu a estação de monta em que foi realizada a pesquisa a estiagem foi prolongada, afetando a produção de pastagem.

Na Propriedade I foram utilizadas 117 novilhas com idade média de 2,7 anos com peso médio de $330 \mathrm{~kg}$ (Grupo I). Na Propriedade II foram utilizadas 143 novilhas Nelore com idade média de 2,5 anos, pesando em média $300 \mathrm{~kg}$ (Grupo II).

Todas as novilhas foram criadas em sistema extensivo com disponibilidade alimentar de capim Brachiaria brizantha, mineralizadas com sal proteinado e água ad libitum. $\mathrm{Na}$ Propriedade I as fêmeas receberam feno no período de seca, antecedendo a estação de monta.

O manejo sanitário das fêmeas constituiu-se de vacinas, vermífugos e brinco mosquicida. Nas Propriedades I e II, o manejo sanitário do rebanho foi composto por vacinas de aftosa e profiláticas como leptospirose, botulismo, rinotraqueíte infecciosa bovina (IBR), diarreia viral bovina (BVD) e clostridiose, o vermífugo utilizado foi à base de Moxidectim $1 \%$. Na Propriedade II foi realizada a administração de vacina antirrábica, devido à localização de alto risco da propriedade.

Nas duas propriedades foi utilizado o mesmo protocolo hormonal: no dia zero (D0) as novilhas receberam um implante intravaginal de progesterona (P4) de quarto uso (Sincrogest ${ }^{\circledR}$ Ouro Fino Saúde Animal, Cravinhos, SP, Brasil) e 2 mg de benzoato de estradiol por via intramuscular (Sincrodiol ${ }^{\circledR}$ Ouro Fino Saúde Animal, Cravinhos, SP, Brasil). Após oito dias (D8) o implante foi retirado e uma segunda dose $(1,0 \mathrm{mg}$, im) de benzoato de estradiol foi aplicado.

Após a remoção dos implantes intravaginais de progesterona, as novilhas retornaram ao pasto e no dia seguinte iniciou-se a observação de cio a cada 12 horas, uma hora no período da manhã e uma hora no período da tarde. Para melhor detecção do cio, rufiões foram utilizados na proporção de um rufião para cada vinte novilhas. As fêmeas que manifestaram cio apresentaram comportamentos distintos como vulva edemaciada, muco brilhante cristalino, o hábito de montar e deixar ser montada. Após observação e detecção de cio, as novilhas que manifestaram cio no período da manhã foram inseminadas no período da tarde do mesmo dia, e as novilhas que manifestaram cio à tarde foram inseminadas no período da manhã do dia seguinte. A observação de estro foi realizada por quinze dias e após este período foram colocados junto das novilhas touros da raça Red Norte, previamente analisados por exames andrológicos para avaliação da fertilidade. Foi utilizado 1 touro para cada 50 novilhas. 
Para determinação da taxa de fertilidade, foi realizado o diagnóstico de gestação cinco meses após a IA pelo método de palpação retal.

Os implantes de progesterona utilizados no estudo eram do modelo em $\mathrm{T}$ e continham $1,0 \mathrm{~g}$ de progesterona. Antes do experimento, os implantes foram utilizados por 3 vezes em vacas multíparas, sendo 8 dias em cada uso. Após a retirada dos implantes, os mesmos eram higienizados com água e detergente desengordurante (CB - 30 T. A., Ouro Fino ${ }^{\circledR}$ Cravinhos, SP, Brasil) e acondicionados em embalagens revestidas internamente com alumínio e posteriormente estocadas em temperatura ambiente.

Para comparação do percentual de novilhas em cio e taxa de fertilidade entre as propriedades I e II foi utilizado o Teste de Qui-quadrado não paramétrico a $5 \%$ de significância, usando o programa SAEG (9.5), (2007).

\section{Resultados e discussão}

Nas Propriedades I e II, a porcentagem de animais em cio foi de $70,94 \%$ e $60,84 \%$, respectivamente, não sendo observadas diferenças entre si (Tabela 1). A manifestação de cio foi mais concentrada nos dois primeiros dias após o término do protocolo (Tabela 2). Os resultados observados no presente estudo demonstraram que a utilização de protocolos hormonais com implante de progesterona de quarto uso foi capaz de induzir a atividade ovariana cíclica em novilhas. Além de contribuir para a redução dos custos, o protocolo hormonal utilizado no presente estudo permitiu que um maior número de fêmeas fosse inseminada na estação de monta, o que possivelmente não aconteceriam se as novilhas não tivessem sido induzidas, aumentando desta forma o número de fêmeas fora da reprodução, resultando em

Tabela 1: Porcentagem de novilhas em cio e taxa de fertilidade de novilhas Nelores, após indução de estro com implantes intravaginais de progesterona de quarto uso

\begin{tabular}{lccc}
\hline & Propriedade I & Propriedade II & \multirow{2}{*}{$p(\%)$} \\
\cline { 2 - 3 } & $\mathrm{n}=117$ & $\mathrm{n}=143$ & \\
\hline Novilhas em cio & $70,94 \%(\mathrm{n}=83)$ a & $60,84 \%(\mathrm{n}=87)$ a & 0,0885 \\
Taxa de Fertilidade & $66,67 \%(\mathrm{n}=78)$ a & $57,34 \%(\mathrm{n}=82)$ a & 0,1242 \\
\hline
\end{tabular}

Letras iguais na mesma linha não apresentam diferenças significativas pelo teste de Qui-quadrado $(p>0,005)$

Tabela 2: Manifestação de cio após a indução com implante de progesterona de quarto uso

\begin{tabular}{lcc}
\hline \multirow{2}{*}{ Dias após indução } & \multicolumn{2}{c}{ Manifestação do cio (\%) } \\
\cline { 2 - 3 } & $\mathrm{n}=117$ & $\mathrm{n}=143$ \\
\hline $1^{\circ} \mathrm{dia}$ & $26,50 \%(31)$ & $27,97 \%(40)$ \\
$2^{\circ} \mathrm{dia}$ & $41,02 \%(48)$ & $29,37 \%(42)$ \\
$3^{\circ} \mathrm{dia}$ & $3,42 \%(4)$ & - \\
$8^{\circ} \mathrm{dia}$ & - & $2,10 \%(3)$ \\
$13^{\circ} \mathrm{dia}$ & - & $1,40 \%(2)$ \\
Total & $70,94 \%(\mathrm{n}=83)$ & $60,84 \%(\mathrm{n}=87)$ \\
\hline
\end{tabular}

prejuízo e descarte de um maior número de novilhas. Azeredo et al. (2007) demonstraram que a progesterona, quando administrada em novilhas, principalmente as pré-púberes, foi capaz de iniciar a atividade estral. Solorzano et al. (2004) demonstraram que $90 \%$ e $93 \%$ de vacas sincronizadas com implantes de progesterona novos ou reutilizados manifestaram sinais de estro após a retirada do implante, quando associado à aplicação de $2 \mathrm{mg}$ de benzoato de estradiol.

Hernández et al. (2008) não encontraram diferença no período de manifestação de estro em vacas Brangus sincronizadas, após a remoção de implante de $\mathrm{P} 4$ novo e reutilizado, que variou de 24 a 80 horas após a remoção do implante.

Os resultados de novilhas em cio e taxa de fertilidade encontrados demonstraram que a utilização do protocolo de indução de cio com implantes de quarto uso aumentou o número de novilhas inseminadas na estação de monta, o mesmo foi encontrado por Busch et al.(2007), que concluíram que a sincronização do estro em novilhas de corte de reposição, submetidas a IATF utilizando protocolo com implante de progesterona mais $\mathrm{GnRH}$ resultou em uma melhor resposta estral e taxa de fertilidade.

Avaliando a manifestação de cio das novilhas após a utilização de implantes de progesterona de quarto uso verificou-se que a maior taxa observada foi no primeiro e segundo dias de observação de cio. Resultados semelhantes foram encontrados por Horn et al. (2001), que reportaram a incidência de cio de novilhas e vacas, sincronizadas com implantes de acetato de medroxi-progesterona e verificaram que houve uma maior concentração de cio nos primeiros três dias de observação e posteriormente uma redução linear significativa ao longo dos seis dias de avaliação na manifestação de estros.

Whisnant e Burns (2002) demonstraram que todas as novilhas tratadas com implantes de progesterona apresentaram comportamento de estro após a retirada e, consequentemente, elevada concentração de progesterona plasmática, consistente com a formação de um corpo lúteo após a ovulação.

Segundo Rasby et al. (1998), trabalhando com novilhas mestiças e protocolo semelhante ao presente estudo, demonstraram que um tratamento de sete dias com progestágenos (implantes intravaginais) já induziria a puberdade nas fêmeas, porém a injeção intramuscular de BE no oitavo dia induziu posteriormente o estro e o aparecimento de corpo lúteo funcional em um maior número de animais, proporcionando, também, sincronia dos sinais de estro, dois a quatro dias após o fim do tratamento, sendo que neste estudo observou se o aparecimento de cio em novilhas Nelore até o $13^{\circ}$ dia após a retirada do implante.

A utilização de protocolos hormonais com implante de progesterona de quarto uso foi capaz de induzir o estro em novilhas, concentrar a manifestação de cio além de diminuir a observação de cio em novilhas (Lima et al. (2003) reportaram que o uso de um dispositivo intravaginal de progesterona mais a aplicação de prostaglandina F2 $\alpha$ (PGF2 $\alpha$ ) resultaram em alta taxa de detecção de cio, retorno ao cio sincronizado e redução do número de dias de observação de cio em vacas nelores solteiras.

A associação de progesterona com o estrógeno tem sido alvo de inúmeros estudos nas últimas décadas, visando 
associar os possíveis benefícios fisiológicos do estrógeno ao seu baixo custo comercial (Vogg et al., 2004). Bragança et al. (2004) demonstraram que no início do tratamento o BE mais P4 provocou atresia dos folículos dominantes presentes e a emergência de uma nova onda folicular após aproximadamente 4, 3 dias da aplicação. Segundo Bridges et al. (1999), esta associação é útil para elevar a taxa de cio e de fertilidade em novilhas próximas à puberdade.

Embora haja redução nos custos com o uso de implantes reutilizados, deve ter o cuidado com a transmissão de doenças sexualmente transmissíveis, cujos estudos ainda são inconclusivos (Thomazi et al., 2009).

Resultados semelhantes da taxa de fertilidade foram encontrados por Cutaia et. al. (2003), que utilizando dispositivo intravaginal de segundo uso em vacas e novilhas, observaram uma taxa de fertilidade de $55 \%$ para vacas e $61,9 \%$ para novilhas.

Resultados diferentes foram encontrados por Baruselli et al. (2006), que reportaram índices de fertilidade de $76 \%$ para novilhas, confirmando que a reutilização de implantes não altera significativamente as taxas de fertilidade.

Os resultados do presente estudo foram semelhantes ao de Rocha et al. (2007), que avaliaram a eficiência da reutilização de um implante intravaginal de progesterona por até quatro vezes, em vacas Nelore submetidas a IATF e observaram taxa de gestação de $50,06 \%, 56,52 \%, 52 \%$ e $31,57 \%$ para implantes novos, reutilizados duas, três e quatro vezes respectivamente, além de observar que não houve diferença significativa entre os grupos de vacas submetidas a IATF e IATF+ Touro.

Peixoto Júnior et al. (2007), utilizando implante intravaginal de terceiro uso, obteve taxa de $75 \%$ de fertilidade em fêmeas de corte e afirmaram que o terceiro uso do implante não diminui a taxa de prenhez, o que viabiliza a implantação destes protocolos nos programas de reprodução.

Segundo Valentim (2004), os resultados da taxa de fertilidade utilizando implantes de quarto uso foram superiores aos implantes novos. Foi observada taxa de fertilidade $25,81 \%$ no grupo de animais tratados com implantes novos e $42,11 \%$ nos que receberam os implantes pela quarta vez consecutiva.

No presente estudo, as taxas de fertilidade foram superiores aos resultados encontrados por Pincinato et al. (2006), que utilizaram implantes intravaginais de progesterona em vacas de corte e leiteiras. Os autores obtiveram 43,3\%, 45\%, 48,3\% e $20 \%$ para os grupos com dispositivo novo de $1 \mathrm{~g} \mathrm{P} 4$, usado com $1 \mathrm{~g} \mathrm{P} 4$,

\section{Referências}

ALMEIDA, A.B.; BERTAN, C.M.; ROSSA, L.A.F.; GASPAR, P.S.; BINELLI, M.; MADUREIRA, E.H. Avaliação da reutilização de implantes auriculares contendo norgestomet associados ao valerato ou ao benzoato de estradiol em vacas nelore inseminadas em tempo fixo. Braz. J. Vet. Re. Ani. Sci .v. 43, n. 4, São Paulo, 2006.

ALMEIDA, J. Avaliação de antiparasitários sobre o perfil enzimático e exame andrológico em Nelore. 2010101 f. - Dissertação (mestrado), Instituto de Veterinária Curso de Pós-Graduação Em Medicina Veterinária, Universidade Federal Rural do Rio de Janeiro - UFRRJ.

AZEREDO, D.M.; ROCHA, D.C.; JOBIM, M.I.M.; MATTOS, R.C.; GREGORY, R.M. Efeito da sincronização e da indução de estros em novilhas sobre a prenhez e o índice de repetição de crias na segunda estação reprodutiva. Ciê. Rur., v. 37, n. 1, 2007. novo com $0,5 \mathrm{~g} \mathrm{P} 4$ e usado com $0,5 \mathrm{~g} \mathrm{P} 4$ respectivamente.

Chesta et al. (2005) não observaram diferença na taxa de fertilidade de animais Hereford com a utilização de dispositivos novos, de segundo e de terceiro uso ( $74 \%$ e $75,3 \%$ respectivamente).

De acordo com os respectivos índices de fertilidade encontrados na Propriedade I $(66,67 \%)$ e na Propriedade II $(57,34 \%)$ é recomendada a utilização de implantes de quarto uso associado ao uso de $\mathrm{BE}$, pois promove um maior número de novilhas ciclando após a indução com o implante, protocolo este também preconizado por Rocha et al. (2007).

O mesmo foi proposto por Rodrigues et al. (2009), que relataram $38,70 \%$ de gestação em novilhas Nelore submetidas à indução de estro e IATF utilizando implante de P4 após três utilizações, e observaram que a utilização de dispositivos para esse fim, contendo menores dosagens de progesterona para novilhas Nelore poderia ser uma alternativa interessante para introduzir esses animais ao rebanho, com antecipação da puberdade.

Semmelmann et al. (2001) obsevaram uma taxa de fertilidade de $20 \%$ trabalhando com novilhas Nelores de 18 meses de idade com peso de $262 \mathrm{~kg}$, resultado inferior ao encontrado neste estudo e este fato pode ser devido ao peso inferior das novilhas utilizadas pelo referido autor.

Embora os animais da Propriedade I tenham passado por um melhor manejo nutricional no período de estiagem precedente à estação de monta, não foi observada diferença estatística no percentual de novilhas em cio e na taxa de fertilidade entre as propriedades analisadas.

\section{Conclusões}

A indução de cio em novilhas Nelore utilizando-se o implante intravaginal de progesterona de quarto uso foi eficaz, considerando o número de animais ciclando após a retirada do dispostivo.

A reutilização de implantes intravaginais de P4 pela quarta vez aumenta o número de novilhas iseminadas na estação reprodutiva além de concentrar a manifestação de cio em dois dias de observação, o que viabiliza a mão de obra.

É possivel afirmar que a associação do uso de implantes intravaginais de P4 pela quarta vez com o repasse de reprodutores é eficiente, além de obter melhores índices de fertilidade em novilhas Nelore submetidas a protocolos de IATF.

BARUSELLI,P.S.; MARQUES,M.O.; NASSER,F.T.; VALENTIM, R.; REIS;E.L. Inseminação artificial em tempo fixo (sem detecção de cio) - (on line). Criar e Plantar, Site: criareplantar.com.br, 2003. Acessado: 20/07/2011.

BARUSELLI, OS; AYRES, H.; SOUZA, A.H.; MARTINS, C.M; GIMENES, L.U; TORRES JÚNIOR, J.R.S. Impacto da IATF na eficiência reprodutiva em bovinos de corte. In: Simpósio Internacional de Reprodução Animal Aplicada, 2., 2006, Londrina, PR. Anais do $2^{\circ}$ Simpósio INTERNACIONAL DE REPRODUÇÃO ANIMAL APLICADA: Faculdade de Medicina Veterinária e Zootecnia, Universidade de São Paulo, v. 1, p. 113-132, 2006.

BRAGANÇA, J.F.M.; GONÇALVES, P.B.D.; BASTOS, G. M.; NEVES J.P.; OLIVEIRA J.F.C.; SIQUEIRA L.C.; BORGES L.F.K.; POMBO R. $D$. Sincronização de estro e ovulação em novilhas de 12 a 14 meses de idade e inseminadas artificialmente com observação de estro e horário pré-fixado. Rev Bras Rep Ani, v. 28, n. 2, p. 73-77, 2004. 
BRASIL. Ministério do Planejamento, Orçamento e Gestão. Instituto Brasileiro de Geografia e Estatística. Produção da Pecuária Municipal. 2011. Disponível em: http://www.ibge.gov.br/home/ presidencia/noticias/noticia_visualiza.php?id_noticia=2002\&id_ pagina=1> Acesso em: março 2012.

BRIDGES, P.J.; LEWIS, P.E.; WAGNER, W.R.; INSKEEP E.K. Follicular growth, estrus and pregnancy after fixed-time insemination in beef cows treated with intravaginal progesterone inserts and estradiol benzoate. Theriogenology, v. 52, p. 573-583, 1999.

BUSCH, D. C.; WILSON, D. J; SCHAFER, D. J; LEITMAN, N.R; HADEN, J. K; ELLERSIECK, M. R; SMITH, M.F. AND PATTERSON D.J.. Comparison of progestin-based estrus synchronization protocols before fixed-time artificial insemination on pregnancy rate in beef heifers. J Anim Sci, v. 85, p. 1933-1939, 2007.

CHESTA, P.; PINCINATO, D.; PENA, D.M.; PERES, L.C.; TRIBULO, R.; BO, G.A. Efecto del tratamiento con DIBR de segundo o tercero uso em protocolos de resincronización de la ovulación y inseminación artificial a tiempo fijo. 6, 2005, Cordoba. Anais do IV SIMPOSIO INTERNACIONAL DE REPRODUCCION ANIMAL. Cordoba, 2005, $1 \mathrm{p}$.

CUTAIA, L.; TRÍBULO, R.; MORENO, D. Pregnancy rates in lactating beef cows treated with progesterone releasing devices, estradiol benzoate and equine chorionic gonadotropin (eCG). Theriogenology, v, 59, p. 216, 2003.

EMERICK, L. L.; DIAS,J.C.; GONÇALVES, P.E.M.; MARTINS'J.A.M.; LEITE, T.G.; ANDRADE'V.J.; Filho Vicente R.V. Aspectos relevantes sobre a puberdade em fêmeas. Rev Bras Reprod Anim, Belo Horizonte, v. 33, n. 1, p. 11-19, 2009.

HERNÁNDEZ, C. W. S. et al. Reutilización de un dispositivo liberador de progesterone (CIDR-B) para sincronizar el estro en un programa de transferencia de embriones bovinos. Téc. Pecu. Méx. v. 46, n. 2, p. 119-135, 2008.

HORN, M.M., GALINA, C.S., MORAES, J.C.F. Padrões de distribuição e métodos de identificação de cios em vacas de corte submetidas a sincronização com progestagéneo/prostaglandina e monta natural. RPCV, v. 96 , n. 539, p. 145-148. (2001).

LIMA, F.S., VASCONCELOS, J.L.M., VILELA, E.R., DIAS, C.C., SÁ FILHO, O.G., MATOS, M.C Protocolos de sincronização de cio em vacas Nelore solteiras - Rev Bras Rep Ani, v. 27, n. 3, p. 442444, 2003.

MARQUES M.O., BARREIROS T.R.R., MAX M.C., SILVA K.C.F., GOMES R.G. \& SENEDA M.M. 2008. IATF: Desafios e soluções para maximizar a eficiência. Timed fixed insemination: challenges and solutions to improve. Ac. Sci. Veter. v. 36, p. 155-160, 2008. (Supl. 2):

MORAES, C.F.; JAUME, C.M.; SOUZA, C.J.H. Controle da reprodução em bovinos de corte. Ministério da Agricultura Pecuária e Abastecimento. Comunicado Técnico 58. Dezembro Bagé, 2005. MOREIRA, R.J.C.; PIRES, MALUF, MADUREIRA, BINELLI, GONÇALVES, LIMA, SUSIN. Uso do protocolo Crestar® em tratamentos utilizando benzoato de estradiol, PGF2 $\alpha$, PMSG e $\mathrm{GnRH}$ para controle do ciclo estral e ovulação em vacas de corte. Braz. J. Vet. Res. Anim. Sci., São Paulo, v. 44, n. 1, p. 56-62, 2007. PEIXOTO JUNIOR, K.C.; ULIAN, C.M.V. Avaliação da taxa de prenhez de vacas tratadas com dispositivos de progesterona reutilizados. Pub. v. 1, n. 4, Ed. 4, Art. 127, ISSN 1982-1263, 2007.
PINCINATO, D.; CUTAIA, L.; PERES, L.C.; BO, G.A Efecto del contenido de progesterona del Dispositivo Intravaginal Bovino DIBR sobre las tasas de prenez obtenidas em vaquillonas cruza Bos indicus inseminadas a tiempo fijo. 2006, Nueva Zelanda. Anais do CONGRESSO MUNDIAL DE REPRODUCCION EM RUMINANTES, Nueva Zelanda, 2006, $1 \mathrm{p}$.

PINTO-NETO, A; SILVA, R. Z; MOTA, M. F; ALBERTON, J. Reutilização de implante intravaginal de progesterona para sincronização de estro em bovinos. Arq Ciênc Vet Zool. UNIPAR, v. 12, n. 2, p. 169-174, 2009.

RASBY, R.J.; DAY, M.L.; JOHNSON, S.K.; KINDER J.E.; LYNCH J.M.; SHORT R.E.; WETTEMANN R.P.; HAFS H.D. Luteal function and estrus in peripubertal beef heifers treated with an intravaginal progesterone releasing device with or without a subsequente injection of estradiol. Therigenology, v. 50, p. 55-63, 1998.

ROCHA, J.M. A., MC RABELO, A. M. HB; SANTOS, B.P.P MACHADO A.C.C BARTOLOMEU A.J.P.;NEVES C.PF; LIMA, A.M.AL; OLIVEIRA. IATF em vacas Nelore: Avaliação de duas doses de eCG e reutilização de implantes intravaginais de progesterona. Med Vet, v. 1, n. 1, p. 40-47, 2007.

ROCHA, M.G; LOBATO, J.F.P. Avaliação do desempenho reprodutivo de novilhas de corte primíparas aos dois anos de idade. Rev Bra Zoot, v. 31, n. 3, p.1388-1395, 2002.

RODRIGUES, L. A. et al. Efeito do implante de progesterona (CIDR e CRONIPRESS MONODOSE) e da aplicação prévia com ultrassonografia na taxa de prenhez de novilhas Nelore (Bos taurus indicus) submetidas a IATF. , 46, 2009, Maringá. Anais da REUNIÃO ANUAL DA SOCIEDADE BRASILEIRA DE ZOOTECNIA. Maringá: SBZ, CDROM.

SEMMELMMANN, C.E.N.; LOBATO, J.P.F.; ROCHA, M.G. Efeito de sistema de alimentação no ganho de peso e desempenho reprodutivo de novilhas Nelores acasaladas aos 17-18 meses. Rev. Soc. Bras Zoot., Viçosa, v. 30, n. 3, p. 835- 834, 2001.

SOLORZANO, C. W. et al. Pregnancy rates after estrus synchronization treatment with new and reused CIDR-B devices. Rep. Fert Devel, v. 16, n. 2, p. 214, 2004.

THOMAZI, S; PINTO-NETO, A; SILVA, R. Z; MOTA; M. F; MELLO, N. M; FONSECA, J. F. Dinâmica ovariana e concentração de progesterona de vacas nelore submetidas a iatf*. Arq. ciênc. Vet. Zool. v. 12, n. 2, p. 135-140, 2009.

VALENTIM, R. Concentrações plasmáticas de progesterona e eficiência reprodutiva de diferentes dispositivos de liberação lenta de progesterona usados em inseminação artificial em tempo fixo. 2004. 88 f. Tese (Doutorado). Programa de Pós-Graduação em Reprodução Animal, Faculdade de Medicina Veterinária e Zootecnia, Universidade de São Paulo.

VOGG, G.; SOUZA, C. J. H.; JAUME, C. M.; MORAES J. C. F. Utilidade do benzoato de estradiol após suplementação com progestágeno na sincronização de cios de novilhas de corte. A Sci Vet, v. 32, p. 41-46, 2004.

WHISNANT, C.S.; BURNS, P.J. Evaluation of steroid microspheres for control of estrus in cows and induction of puberty in heifers. Theriogenology, v. 58, n. 6, p. 1229-1236, 2002. 